\title{
Simvastatin suppresses homocysteine-induced apoptosis in endothelial cells: roles of caspase-3, CIAP-1 and CIAP-2
}

\author{
Zhihong $\mathrm{Xu}^{1}$, Guoping $\mathrm{Lu}^{2}$ and Fang $\mathrm{Wu}^{1}$
}

The present study addresses how homocysteine (Hcy) induces endothelial apoptosis and how simvastatin antagonizes these pro-apoptotic effects of Hcy. Human umbilical vein endothelial cells (HUVECs) were treated with Hcy for $24 \mathrm{~h}$, in the presence or absence of simvastatin. Cell apoptosis was evaluated by Annexin V staining and flow cytometry, as well as transferasemediated dUTP-biotin nick end labeling (TUNEL). The mRNA and protein levels of caspase-3, cellular inhibitor of apoptosis (cIAP)-1 and -2 were analyzed by real-time polymerase chain reaction (RT-PCR) and western blotting, respectively. Treatment with both low $\left(0.05 \mathrm{mmol}^{-1}\right)$ and high $\left(0.3 \mathrm{mmol} \mathrm{I}^{-1}\right)$ concentrations of Hcy induced apoptosis in HUVECs which was accompanied by an increased level of caspase-3 expression and activation, together with decreased cIAP-1 and cIAP-2 levels. Conversely, simvastatin upregulated c-IAP1 and c-IAP2 expression, while attenuating Hcy-induced apoptosis and caspase-3 activation. Hcy may induce HUVEC apoptosis through a pathway involving caspase-3. This induction can be partially antagonized by simvastatin, possibly through upregulation of cIAP-1 and cIAP-2.

Hypertension Research (2009) 32, 375-380; doi:10.1038/hr.2009.24; published online 3 April 2009

Keywords: homocysteine; simvastatin; apoptosis; caspase; clAP

\section{INTRODUCTION}

Increases in plasma homocysteine (Hcy) levels cause hyperhomocysteinemia. Recently, through etiological studies and clinical research, hyperhomocysteinemia has been recognized as an independent risk factor of cardiovascular diseases such as atherosclerosis and myocardial infarction. ${ }^{1}$ Using animal models, Hcy was shown to lead to the formation of atheromatous plaques and thrombi by damaging the vascular endothelium. Apoptotic endothelial cells were observed in atheromatous plaques. In vitro, Hcy treatment causes significant apoptosis of endothelial cells in tissue culture studies, thereby indicating that Hcy-induced endothelial cell apoptosis may play an important role in the onset and development of atherosclerosis. ${ }^{2}$

Apoptotic signals are transduced primarily through caspasemediated signaling pathways. The principal process is the activation of the rate-limiting molecule, caspase- 3 . These signal transduction pathways are regulated by multiple regulatory factors, including positive regulators and negative regulators. The cellular inhibitor of apoptosis protein-1 (cIAP-1 or HIAP-2/hMIHB) and cellular inhibitor of apoptosis protein-2 (cIAP-2 or HIAP-1/hMIHC) are endogenous negative regulators of these apoptotic pathways. In addition, they can block the activation of caspases. ${ }^{3}$

In this study, we examined the possible involvement of caspases in Hcy-induced apoptosis of endothelial cells. Furthermore, we explored the antagonistic effect of simvastatin and the role of cIAPs in this apoptotic induction.

\section{METHODS}

Chemicals and reagents

Hcy was purchased from Sigma (St Louis, MO, USA) and Simvastatin was from Merck (Whitehouse Station, NJ, USA). Cell culture medium, fetal bovine serum (FBS), trypsin and collagenase were obtained from Invitrogen (Carlsbad, CA, USA). Antibodies against caspase-3, cIAP-1, cIAP-2, $\beta$-actin and horseradish peroxidase (HRP)-labeled secondary antibodies were from Santa Cruz (Beverly, MA, USA). Enhanced chemiluminescent detection (ECL) reagents and FITCannexin $\mathrm{V}$ were from BD Biosciences (San Jose, CA, USA). The terminal deoxynucleotidyl transferase-mediated dUTP-biotin nick end labeling (TUNEL) detection kit was from Roche (Basel, Switzerland).

Isolation and culture of human umbilical vein endothelial cells (HUVECs)

Cords were washed with sterile Hank's balanced salt solution three times and then digested with $1 \%$ collagenase at $37^{\circ} \mathrm{C}$ for $15 \mathrm{~min}$. After centrifugation, the cells were resuspended in M199 medium with $10 \% \mathrm{FBS}, 100 \mathrm{IU} \mathrm{ml}^{-1}$ penicillin and $100 \mathrm{IU} \mathrm{ml}^{-1}$ streptomycin, and they were maintained at $37^{\circ} \mathrm{C}$ in a $5 \% \mathrm{CO}_{2}$ incubator. The medium was changed $24 \mathrm{~h}$ later to remove the unattached cells. The cells were digested with trypsin-EDTA and subcultured when they reached $80 \%$ confluence. HUVECs positive for Factor VIII were used between passage 2 and passage 10 in all further experiments.

${ }^{1}$ Department of Geriatrics, Ruijin Hospital, Shanghai Jiaotong University, Shanghai, China and ²Department of Cardiology, Ruijin Hospital, Shanghai Jiaotong University, Shanghai, China

Correspondence: Professor F Wu, Department of Geriatrics, Ruijin Hospital, Shanghai Jiaotong University, No. 197, Rui Jin Er Road, Shanghai 200025, PR China. E-mail: Dr.WuFang@hotmail.com

Received 4 November 2008; revised 21 January 2009; accepted 8 February 2009; published online 3 April 2009 


\section{Cell treatment}

HUVECs were maintained in M199 medium containing FBS, penicillin and streptomycin. Before drug treatment, cells were starved in serum-free medium for $24 \mathrm{~h}$. They were then treated for $24 \mathrm{~h}$ with different concentrations of Hcy and simvastatin in complete medium. The treatments were performed in triplicate, in more than three independent experiments.

\section{Fluorescence-activated cell sorting (FACS)}

After treatment, the cells were collected and washed with ice-cold PBS three times. Then, $1 \times 10^{6}$ cells were resuspended in $200 \mu$ of binding buffer and incubated together with $10 \mu \mathrm{l}$ of FITC-Annexin V $\left(200 \mu \mathrm{g} \mathrm{ml}^{-1}\right)$ at room temperature for $15 \mathrm{~min}$ in a foil-covered tube. After incubation, the reaction solution was mixed with $500 \mu \mathrm{l}$ of buffer and then analyzed in a FACS Caliber cell sorter (Becton Dickinson, Franklin Lakes, NJ, USA). ${ }^{4}$

\section{Terminal deoxynucleotidyl transferase-mediated biotin-dUTP nick end labeling}

These experiments were performed according to the manufacturer's protocol (Roche). Briefly, the cells were washed with ice-cold PBS and fixed with $4 \%$ paraformaldehyde at room temperature for $1 \mathrm{~h}$. After washing with PBS three times, the cells were incubated for $2 \mathrm{~min}$ on ice in a permeabilization buffer ( $0.1 \%$ Triton X-100 in $0.1 \%$ sodium citrate solution). After three washes with PBS, the cells were air-dried and incubated with $50 \mu$ of TUNEL reaction buffer at $37^{\circ} \mathrm{C}$ for $1 \mathrm{~h}$ in a foil-covered wet box. Then the cells were washed with PBS three times and photographed under a Zeiss 510 laser scanning confocal fluorescence microscope (Carl Zeiss MicroImaging, Göttingen, Germany). The average of 10 random views of a coverslip was assessed as apoptosis index (AI) and counted for each position cell ratio (apoptosis), and experiments were repeated on three independent sets.

\section{RNA extraction and RT-PCR}

The total cellular RNA was extracted using Trizol reagents according to the manufacturer's protocol. The quantity of RNA samples was determined by spectral absorption at 260 and $280 \mathrm{~nm}$. The quality of RNA was further examined by agarose gel electrophoresis. The PCR primers used in this study were the following: caspase- 3 (PCR product: $885 \mathrm{bp}$ ), 5'-GCTAGCTAGCAT GGAGAACACTGAAAACTCAGTG-3' (caspase3-f) and 5'-GCTACTCGAGG TGATAAAAATAGAGTTCTTTTGTG-3' (caspase3-r); cIAP-1 (280 bp), 5'-TGA GCATGCAGACACATGC- $3^{\prime}$ (cIAP-1-f) and $5^{\prime}$-TGACGGATGAACTCCTGT CC-3' (cIAP-1-r); cIAP-2 (408 bp), 5'-AATGGAAGATAGCACGAT-3' (cIAP-2-f) and $5^{\prime}$-AGAAAGGCTGGAGTAAGA- $3^{\prime}$ (CIAP-2-r). The RT-PCR was performed using a kit from Promega (Madison, WI, USA) and $\beta$-actin was used as an internal standard. The PCR products were separated on $1.2 \%$ agarose gel and stained with ethidium bromide (EB). The gel images were taken using the ImageScanner (GE Healthcare, Piscataway, NJ, USA) and analyzed using ImageQuant software (GE Healthcare).

\section{Western blotting}

The cells were collected and lysed in SDS loading buffer. After boiling for $5 \mathrm{~min}$, total cell lysates from each sample were resolved by SDS-PAGE and transferred electrophoretically to polyvinylidene fluoride membranes. The resulting membranes were blocked using 15\% non-fat milk in TBST (10 mм Tris-HCl, pH 7.4, $150 \mathrm{~mm} \mathrm{NaCl}, 0.05 \%$ Tween-20), and hybridized with the indicated primary antibodies overnight at $4{ }^{\circ} \mathrm{C}$. After incubation with the appropriate HRPconjugated secondary antibody, the immune complexes were detected using ECL reagents and autoradiography. Protein quantification was performed using ImageQuant software, with $\beta$-actin as internal standard, as described earlier. ${ }^{5}$

\section{Statistics}

Data are expressed as mean \pm s.d. of at least three independent experiments, except where otherwise indicated. The Q test was used for statistical analysis of paired comparisons among multiple groups. The chi-square $\left(\chi^{2}\right)$ test was used to analyze differences between groups. All statistical analysis was carried out using SPSS 10.0 software (SPSS Inc., Chicago, IL, USA). Differences were considered statistically significant at $P<0.05$.

\section{RESULTS}

Hcy induces apoptosis of HUVECs

To detect the apoptosis of HUVECs after treatment with Hcy and simvastatin, FITC-Annexin V staining followed by FACS analysis was carried out. As shown in Figure 1a, the control-treated HUVECs showed a spontaneous apoptosis rate of approximately $0.5 \%$, indicating that the HUVECs were of good quality and showed a low baseline rate of apoptosis. Following $0.05 \mathrm{~mm}$ Hcy treatment, however, the proportion of apoptotic cells increased to $12 \%$ (Figure $1 \mathrm{~b}$ ). When the concentration of Hcy reached $0.3 \mathrm{~mm}$, the proportion of HUVECs that were apoptotic further increased to approximately 30\% (Figure 1c). These results are consistent with earlier studies, demonstrating that Hcy treatment can lead to cell apoptosis. Interestingly, when simvastatin $(10 \mu \mathrm{M})$ was applied to HUVECs together with $0.3 \mathrm{~mm} \mathrm{Hcy}$, the apoptotic proportion fell to $10 \%$ (Figure 1d). This indicates that simvastatin can antagonize the pro-apoptotic effect of Hcy.

Next, the TUNEL method was used to confirm the apoptotic effects of Hcy. When a cell undergoes apoptosis, intracellular endonucleases are activated and they cleave the chromosomal DNA between the nucleosomes, leading to DNA fragmentation, which is one of the defining characteristics of apoptosis. The cleaved DNA termini in the nuclei can be recognized and labeled by terminal deoxynucleotidyl transferase in situ. In this way, the TUNEL method allows ready detection of DNA fragmentation. These results are shown in Figure 2. After Hcy treatment, the contracted and condensed HUVEC nuclei were labeled specifically in situ, and they therefore showed yellowgreen fluorescence (Figure 2, arrowhead). Meanwhile, we found that co-administration of simvastatin significantly decreases the number of apoptotic cells caused by Hcy (data not shown).

\section{The mRNA levels of caspase-3, cIAP-1 and cIAP-2}

RT-PCR was used to detect the mRNA expression levels of caspase-3, CIAP-1 and cIAP-2 genes. The PCR products were separated on agarose gels and stained with EB. After photography, the intensity of the individual bands was analyzed and normalized to the intensity of $\beta$-actin in order to quantify the expression levels. Relative mRNA abundance was calculated by dividing the intensity of the gene of interest by the intensity of the $\beta$-actin signal. As shown in Figure 3 and Table 1, the relative mRNA level of caspase- 3 was 0.51 in the control group. It increased to $0.98(P<0.05)$ after treatment with $0.05 \mathrm{~mm}$ Hcy and to $2.54(P<0.05)$ after treatment with $0.3 \mathrm{~mm}$ Hcy, suggesting that Hcy stimulation upregulates caspase-3 expression. On the contrary, the relative cIAP-1 and cIAP- 2 mRNA levels of the control group were as high as 2.21 and 2.02, respectively. However, they decreased to $0.84(P<0.05)$ and $0.62(P<0.05)$ following treatment with $0.3 \mathrm{~mm}$ Hcy. Treatment with $0.05 \mathrm{~mm}$ Hcy also downregulated cIAP-1 and cIAP-2 expression, the differences were not statistically significant compared with control cells. These results suggest that Hcy stimulation upregulates caspase- 3 and downregulates expression of cIAP-1 and CIAP-2.

On the other hand, in cells treated with both $0.3 \mathrm{~mm}$ Hcy and $10 \mu \mathrm{M}$ simvastatin, the relative caspase-3 levels were significantly lower $(P<0.05)$, and the levels of cIAP-1 and cIAP-2 were significantly higher $(P<0.05)$, than in cells treated with $0.3 \mathrm{~mm}$ Hcy alone. These results show that simvastatin antagonizes the effects of Hcy in regulating expression of caspase-3, cIAP-1 and cIAP-2.

\section{Protein levels of caspase-3, cIAP-1 and cIAP-2}

The protein levels of caspase- 3 , cIAP-1 and cIAP- 2 were assessed by Western blotting and quantified using $\beta$-actin as an internal standard. In the cells treated with $0.3 \mathrm{~mm}$ Hcy, the level of activated caspase- 3 

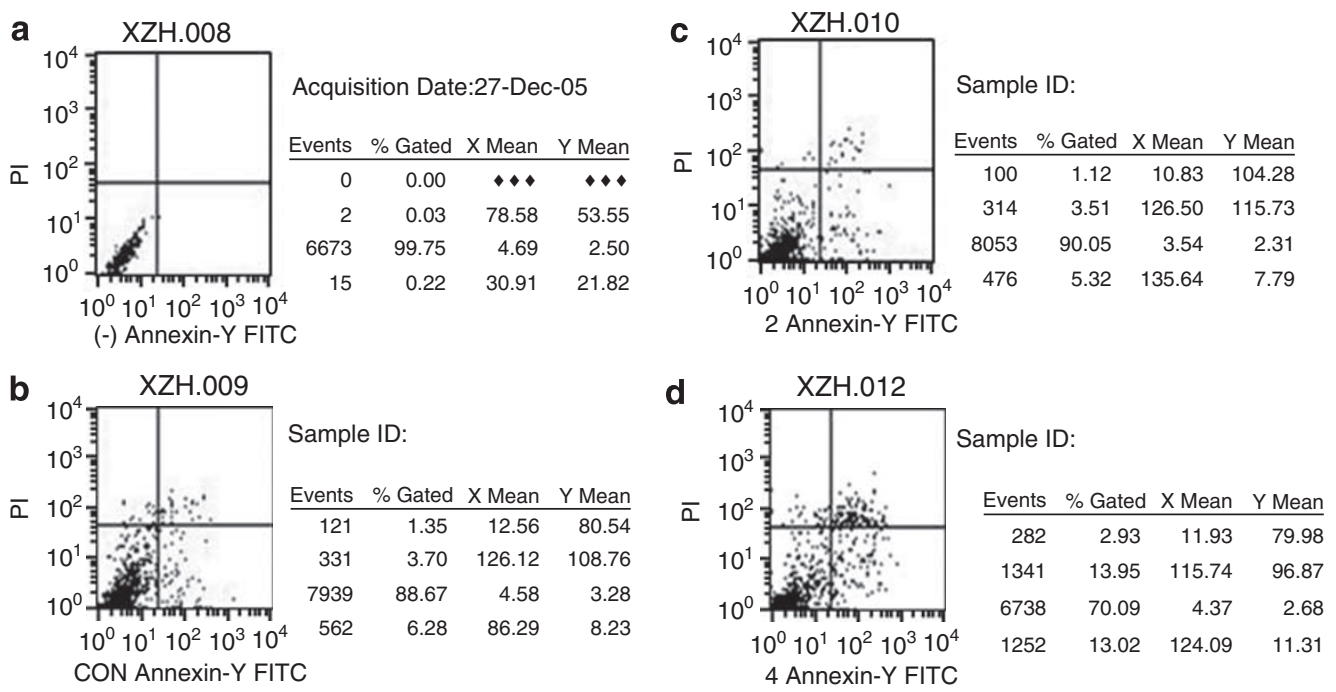

Figure 1 Human umbilical vein endothelial cell (HUVEC) apoptosis detected using annexin V staining and fluorescence-activated cell sorting (FACS). HUVECs were mock-treated (a) or treated with $0.05 \mathrm{~mm} \mathrm{Hcy} \mathrm{(b),} 0.3 \mathrm{~mm} \mathrm{Hcy} \mathrm{(c)} \mathrm{or} 0.3 \mathrm{~mm} \mathrm{Hcy} \mathrm{in} \mathrm{addition} \mathrm{to} 10 \mu \mathrm{m}$ simvastatin (d) for $24 \mathrm{~h}$, and then apoptosis was assessed by annexin V staining and flow cytometry. The dot plots display annexin V staining ( $x$ axis) and PI staining ( $y$ axis); PI is a nucleic acid stain that binds to the DNA within the cell after the membrane breaks down. Annexin V-positive cells are in the upper-right and lower-right quadrants, and PI-positive cells are in the upper-right and upper-left quadrants. Cells in different states are distributed in the plots as follows: the upper-left quadrant contains nuclear debris; the upper-right quadrant, late apoptotic cells; the lower-left quadrant, healthy, viable cells; and the lower-right quadrant, cells in the early stages of apoptosis. Results shown are representative of at least three independent experiments. (a) The control-treated HUVECs showed a spontaneous apoptosis rate of approximately $0.5 \%$. (b) Following $0.05 \mathrm{~mm}$ Hcy treatment, the proportion of apoptotic cells were increased to $12 \%$. (c) The proportion of HUVECs that were apoptotic further increased to approximately $30 \%$. (d) When simvastatin ( $10 \mu \mathrm{m}$ ) was applied to HUVECs together with $0.3 \mathrm{~mm}$ Hcy, the apoptotic proportion fell to $10 \%$.

protein was significantly higher (Figures $4 \mathrm{a}$ and $\mathrm{b} ; P<0.05$ ), and the levels of cIAP-1 and cIAP-2 proteins significantly lower (Figures $4 \mathrm{a}, \mathrm{c}$, and $\mathrm{d} ; P<0.05)$, than in control cells. Thus, Hcy treatment induced similar changes in the levels of both protein and mRNA for caspase- 3 , cIAP-1 and cIAP-2. These results confirm that Hcy induces upregulation and activation of caspase-3, and down-regulation of cIAP-1 and cIAP-2. Similar trends were observed when cells were treated with $0.05 \mathrm{~mm}$ Hcy, but the differences between the treated cells and control cells did not reach statistical significance.

In HUVECs treated with both simvastatin and $0.3 \mathrm{~mm} \mathrm{Hcy}$, the level of activated capase- 3 was significantly lower $(P<0.05)$, and the levels of cIAP-1 and cIAP-2 significantly higher $(P<0.05)$, than in HUVECs treated with only $0.3 \mathrm{~mm} \mathrm{Hcy}$. These changes in protein levels also mimic the changes in mRNA levels, further suggesting that simvastatin protects HUVECs from apoptosis by antagonizing Hcy-induced upregulation of caspase- 3 and downregulation of cIAP-1 and cIAP-2.

\section{DISCUSSION}

In recent years, an increasing number of clinical and laboratory studies have shown that hyperhomocysteinemia is a risk factor for atherosclerosis (AS), and for arterial and venous thrombosis. Furthermore, serum Hcy levels affect vasculopathy in a dose-dependent manner. In a meta-analysis involving 5073 ischemic heart disease events and 1113 stroke events, it is found that when serum Hcy levels increase by $3 \mu \mathrm{M}$ the rate of ischemic heart disease increases by $11 \%$, and the rate of apoplexia climbs to $19 \%{ }^{6}$ Therefore, prevention and therapeutic management of hyperhomocysteinemia has gained a significant amount of attention from researchers recently.

Endothelial cells can be detected in patients with severe hyperhomocysteinemia. In studies with animal models, Hcy levels have been shown to lead to multiple pathological changes, including shedding of the arterial endothelium, local thrombosis and smooth muscle cell proliferation. The toxicity of Hcy to vascular endothelial cells has also been demonstrated through in vitro studies. Hcy in serum may be able to auto-oxidize to form disulfide homocystin (Hcy-SS-Hcy), homocysteine-cysteine mixed disulfide and Hcy-thiolactone during oxidation caused by such reactive oxygen species (ROS) such as peroxide $\left(\mathrm{H}_{2} \mathrm{O}_{2}\right)$, hydroxyl radical $\left(-\mathrm{OH}^{\bullet-}\right)$ and superoxide $\left(\mathrm{O}_{2}^{\bullet-}\right)$. These ROS, particularly $-\mathrm{OH}^{\bullet-}$, can react with the unsaturated fatty acids in the plasma membrane and initiate the chain reaction that leads to peroxidation of plasma membrane lipids. These reactions compromise the integrity of the plasma membrane and eventually cause functional alterations in endothelial cells, which lead to cellular apoptosis and the shedding of these cells. Therefore, it has been hypothesized that the endothelial damage caused by $\mathrm{Hcy}$ is one of the reasons why hyperhomocysteinemic patients usually show early onset of cardiovascular diseases. ${ }^{7}$

Apoptosis is a form of cell-autonomous death involved in multiple processes, such as embryonic development, cell differentiation and pathological events. An enormous number of molecular and cellular changes are associated with apoptosis. The regulation of apoptosis may also occur on multiple levels, including signal transduction, apoptotic gene expression and activity of apoptotic enzymes. For this reason, a multitude of detection methods have been developed to target the different stages of apoptosis. In the TUNEL method, the cleaved DNA ends generated by apoptotic endonucleases are detected by fluorescent labeling, which ensures high specificity of the signal. On the other hand, phosphatidyl serine in the plasma membrane flips into the outer leaflet of the phospholipid bilayer in the early stages of apoptosis. By detecting phosphatidyl serine using annexin $\mathrm{V}$ staining, cells in the early stages of apoptosis can easily be observed. Using this method, we found that treatment with $0.05 \mathrm{~mm}$ Hcy causes changes in 
a

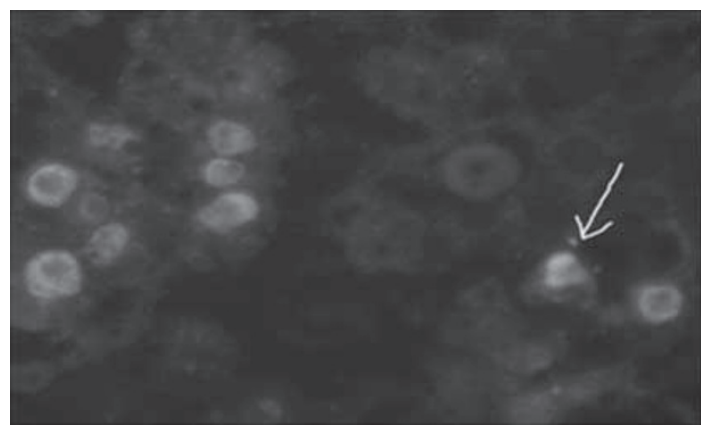

b

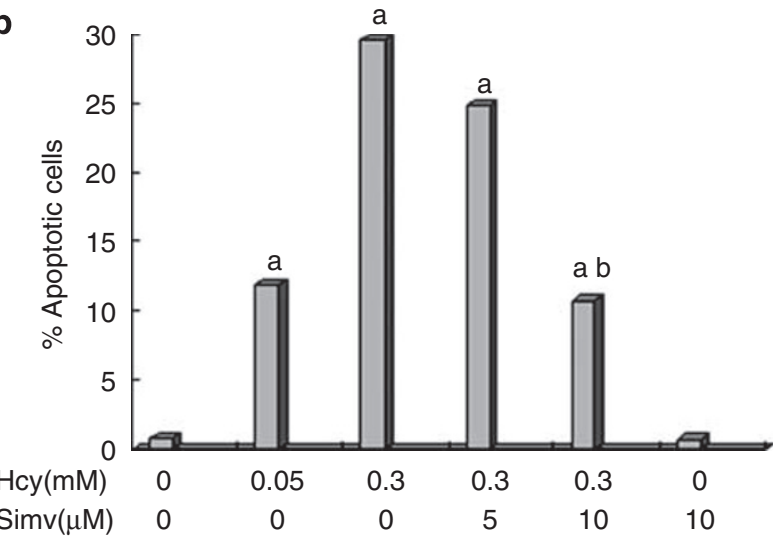

Figure 2 Human umbilical vein endothelial cell (HUVEC) apoptosis detected using transferase-mediated dUTP-biotin nick end labeling (TUNEL). The arrow indicates a TUNEL-positive cell. Hcy treatment induced apoptosis index (AI) increase in HUVECs in a dose-dependent manner. In the control group, Al was $0.74 \pm 0.12 ; 0.3 \mathrm{mmol}^{-1}$ Hcy treatment was $29.61 \pm 0.55$ $(P<0.05)$. Quantitative analysis demonstrated that simvastatin $\left(10 \mu \mathrm{mol} \mathrm{I}^{-1}\right)$ significantly inhibited Hcy-induced apoptosis in HUVECs $(P<0.05$ versus $0.3 \mathrm{mmol} / \mathrm{l}$ Hcy treatment) ( $n=10, x \pm s, \%)$. Values are mean \pm s.e., $\%$ and $n=10$; (a), $P<0.05$ showed significant difference with control treatment; (b), $P<0.01$ showed significant difference with $0.3 \mathrm{mmoll}^{-1} \mathrm{Hcy}$ treatment.

the cell membrane of HUVECs, suggesting that even a low concentration of Hcy induces cellular apoptosis.

Kokame et al. ${ }^{8}$ reported that gene expression of HUVEC was changed by $1-10 \mathrm{mmoll}^{-1}$ Hcy. However, $0.05 \mathrm{mmoll}^{-1}$ Hcy caused cell membrane change in our experiments. Although apoptosis can be induced by multiple signals, the caspase-mediated pathway is usually the general route for them. The caspase pathway plays an important role in the apoptosis signal transduction. The caspase family is a collection of proteases, which is also called the ICE/CED-3 family. They are directly involved in the triggering and signal transduction of the apoptotic cascade. ${ }^{9}$ One of the caspases, caspase- 3 , holds a central position in the apoptotic cascade. Generally, the core reaction is the activation of executive caspase, caspase-3, which cleaves the inhibitor of endonuclease and thereby activates it to cut the DNA. In the cytosol, cyt c binds to and activates Apaf-1, which then activates caspase- 9 and caspase- 3 to induce the apoptosis; FADD is recruited by the Fas receptor, which causes caspase- 8 oligomerization and activates caspase-3, resulting in apoptosis. Our western blotting results showed that the expression of caspase- 3 in $0.3 \mathrm{mmoll}^{-1}$ HUVEC was increased, which formed a shadow band at $17 \mathrm{kDa}$. However, the expression of caspase- 3 was significantly inhibited by $10 \mu \mathrm{m}$ simvastatin in the simvastatin-treated group.

Multiple negative regulators exist that inhibit caspases, and the IAP family is a class of apoptosis inhibitors. Members of this family possess a

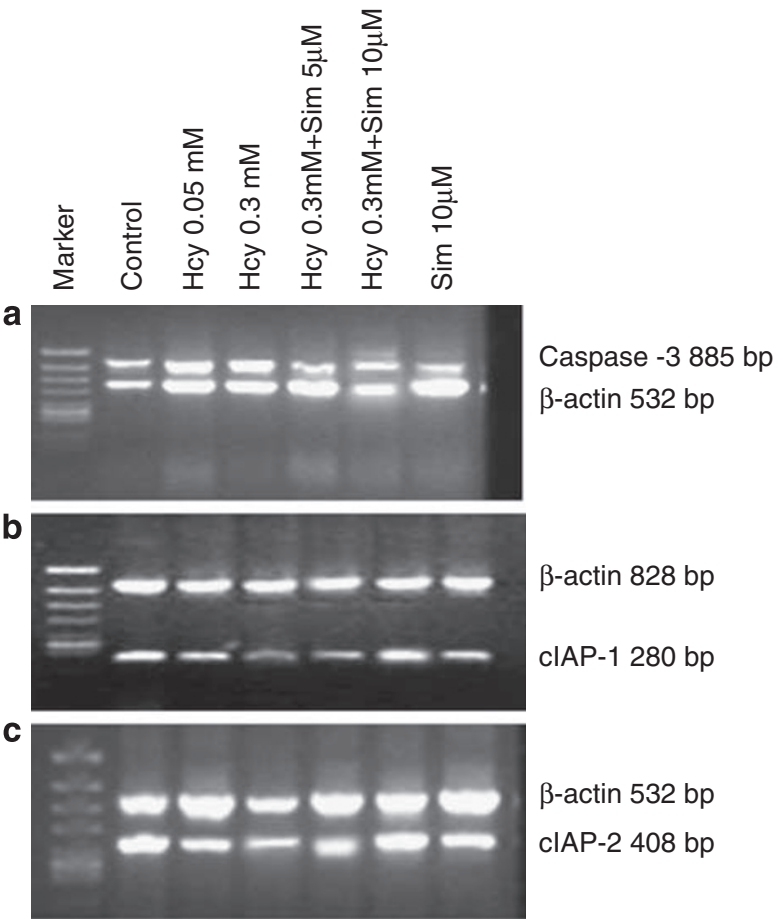

Figure 3 Effects of different treatments on the expression levels of caspase3 , cellular inhibitor of apoptosis protein (cIAP)-1 and -2 in HUVECs, as detected by RT-PCR. The mRNA expression levels of Caspase-3, clAP-1 and ClAP-2 genes were detected using the RT-PCR. The polymerase chain reaction (PCR) products were separated on agarose gels and stained with ethidium bromide. After photography, the intensity of the individual bands was analyzed and normalized to the intensity of $\beta$-actin in order to quantify the expression levels. (Relative mRNA abundance was calculated by dividing the intensity of the gene of interest by the intensity of the $\beta$-actin signal.) Different treatments are labeled in (a), and the same pattern is used in panels (b) and (c). Results shown are representative of at least three independent experiments.

Table 1 Relative mRNA levels of caspase-3, cIAP-1 and cIAP-2 in HUVECs

\begin{tabular}{llll}
\hline Groups & Caspase-3 & \multicolumn{1}{c}{ clAP-1 } & \multicolumn{1}{c}{ clAP-2 } \\
\hline Control & $0.51 \pm 0.04$ & $2.21 \pm 0.10$ & $2.02 \pm 0.12$ \\
Hcy $(0.05 \mathrm{~mm})$ & $0.98 \pm 0.05^{\mathrm{a}}$ & $1.98 \pm 0.06$ & $1.89 \pm 0.10$ \\
Hcy $(0.3 \mathrm{~mm})$ & $2.54 \pm 0.06^{\mathrm{a}}$ & $0.84 \pm 0.05^{\mathrm{a}}$ & $0.62 \pm 0.06^{\mathrm{a}}$ \\
Hcy $(0.3 \mathrm{~mm})+\operatorname{simvastatin}(5 \mu \mathrm{m})$ & $1.67 \pm 0.02$ & $0.96 \pm 0.01$ & $0.68 \pm 0.05$ \\
Hcy $(0.3 \mathrm{~mm})+\operatorname{simvastatin}(10 \mu \mathrm{m})$ & $0.60 \pm 0.03^{\mathrm{b}}$ & $1.99 \pm 0.02^{\mathrm{b}}$ & $2.00 \pm 0.04^{\mathrm{b}}$ \\
Simvastatin $(10 \mu \mathrm{m})$ & $0.57 \pm 0.06$ & $2.12 \pm 0.03$ & $1.99 \pm 0.05$
\end{tabular}

Abbreviations: Hcy, homocysteine; HUVECs, human umbilical vein endothelial cells.

The results shown in Figure 3 were quantified and represented as the intensity of the target mRNA divided by the intensity of $\beta$-actin. Values are shown as mean \pm s.e.m. of at least three independent experiments.

Independent experiments.
alndicated significant difference compared to the control cells $(P<0.05)$.

${ }^{b}$ Indicated significant difference compared to the cells treated with $3 \mathrm{~mm} \mathrm{Hcy}(P<0.05)$.

highly conserved repetitive signature domain, originally described as a baculoviral apoptotic inhibitor. IAPs directly inhibit apoptosis by preventing caspase- $3,-9$ and -7 from initiating and executing the caspase cascade. So far, seven members of the mammalian IAP family have been identified, including cIAP-1, cIAP-2, XIAP, apollon, ILP2, livin and survivin. ${ }^{10}$ Evidence showed that cIAP-1 and cIAP-2 were highly correlated with cell apoptosis, therefore we studied them in our experiments. $^{11,12}$ In our experiment, we also observed two caspase-3 

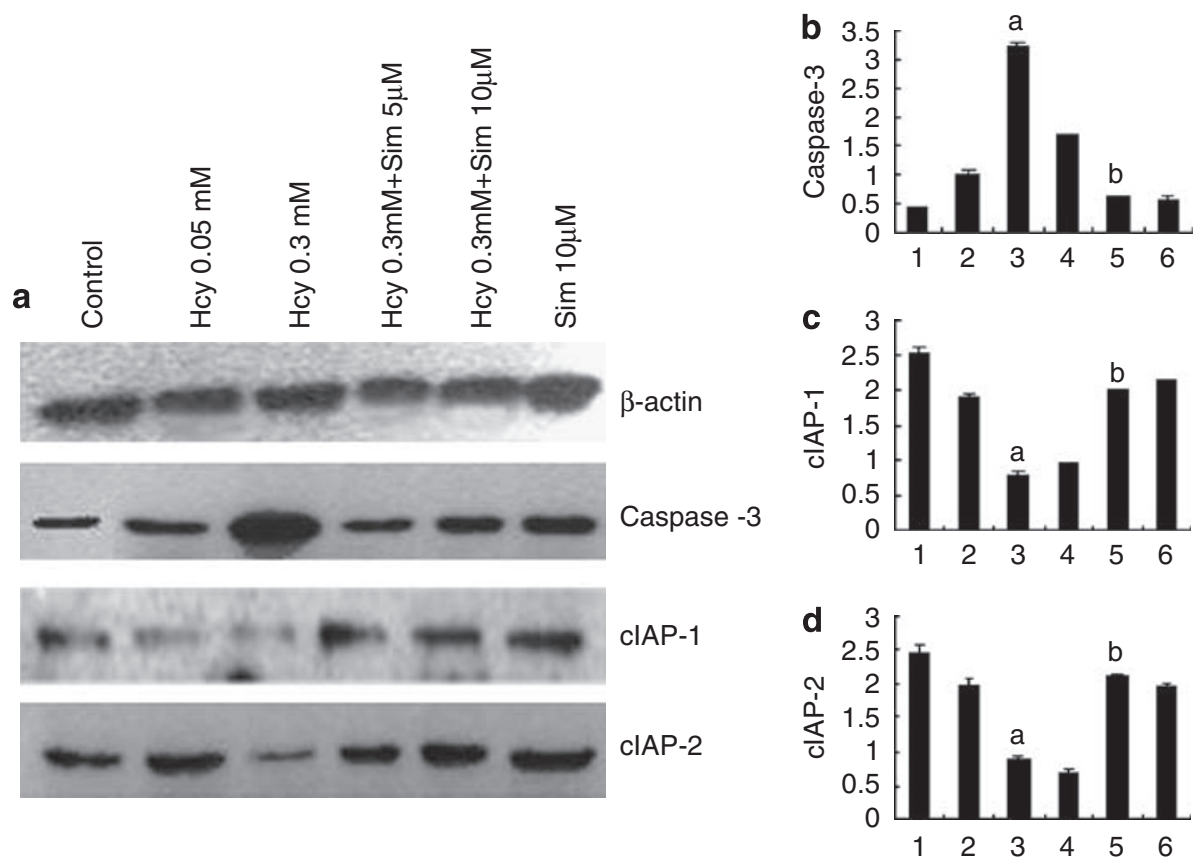

Figure 4 Effects of different treatments on the protein levels of caspase-3, cellular inhibitor of apoptosis protein (clAP)- 1 and -2 detected by Western blotting. (a) Proteins were separated by sodium dodecyl sulfate-polyacrylamide gel electrophoresis (SDS-PAGE) and blotted with the indicated antibodies (1:1000). The different groups are as follows: lane 1, control treated; lane 2, $0.05 \mathrm{~mm} \mathrm{Hcy;} \mathrm{lane} \mathrm{3,} 0.3 \mathrm{~mm} \mathrm{Hcy}$; lane $4,0.3 \mathrm{~mm} \mathrm{Hcy}+5 \mu \mathrm{m}$ simvastatin; lane 5, $0.3 \mathrm{~mm} \mathrm{Hcy}+10 \mu \mathrm{m}$ simvastatin; lane $6,10 \mu \mathrm{m}$ simvastatin. Results shown are representative of at least three independent experiments. (b-d) Quantification of the levels of activated caspase-3 (b), clAP-1 (c) and clAP-2 proteins (d). The order of the treatments is the same as that in (a). Results were expressed as the intensity of the target protein divided by the intensity of $\beta$-actin; values are mean \pm s.e.m. of at least three independent experiments (a, $P<0.05$ compared to group $1 ; \mathbf{b}, P<0.05$ compared to group 3 ).

inhibitory enzymes and found that the expressions of cIAP-1 and CIAP2 were enhanced in the $10 \mu \mathrm{m}$ simvastatin-treated group. Hcy treatment caused an upregulation of caspase- 3 mRNA expression and activated caspase- 3 protein. In addition, it downregulated mRNA and protein levels of cIAP-1 and cIAP-2. These results suggest that Hcy may induce HUVEC apoptosis through the caspase pathway. However, Mercie et al. have reported that Hcy-thiolactone may induce endothelial cell apoptosis through a non-caspase pathway. Therefore, there may be multiple pathways through which Hcy and its derivatives induce apoptosis. ${ }^{11}$

HMG-CoA reductase inhibitors (statins) are effective in treating coronary heart diseases and related diseases, as shown by evidencebased medicine. Besides regulating serum lipid levels, statins can improve inflammation, protect the endothelium, downregulate matrix metalloproteases (MMPs) and stabilize AS plaques. We reasoned that these effects of statins would allow them to protect against the vascular toxicity of Hcy. Although there is evidence that statins have apoptotic and growth-inhibitory effects on cells, including endothelial cells in vitro and in vivo, ${ }^{13-16}$ statins have different effects on endothelial cell apoptosis and growth inhibition with different doses or different types of statins. For instance, small doses of statins inhibited lymphocyte activation and decreased the proinflammatory cytokine production. Simvastatin is one form of statins with high fat solubility. Therefore, it can accumulate to higher levels in tissues (including endothelial cells) than in blood as a result of passive diffusion. However, whether simvastatin antagonizes Hcy-induced HUVEC apoptosis has not been reported. We demonstrate in this report that $10 \mu \mathrm{M}$ simvastatin effectively inhibits Hcy-induced caspase-3 activation and HUVEC apoptosis, possibly by upregulating CIAP-1 and cIAP-2. This work has identified the ability of a statin to protect against the endothelial toxicity of Hcy, thereby providing a new strategy for treating homocysteinemia and extending the therapeutic scope of statins.

\section{ACKNOWLEDGEMENTS}

This research was supported by a grant from Ruijin Hospital, Shanghai Jiaotong University.

\section{CONFLICT OF INTEREST}

The authors declare no conflict of interest.

1 Au-Yeung KK, Woo CW, Sung FL, Yip JC, Siow YL, O K. Hyperhomocysteinemia activates nuclear factor-kB in endothelial cells via oxidative stress. Circ Res 2004; 94: 28-36.

2 Chambers JC, Obeid OA, Kooner JS. Physiological increments in plasma homocysteine induce vascular endothelial dysfunction in normal human subjects. Arterioscler Thromb Vasc Biol 1999; 19: 2922-2927.

3 Roy N, Deveraux QL, Takahashi R, Salvesen GS, Reed JC. The C-IAP-1 and C-IAP-2 proteins are direct inhibitors of specific caspases. EMBO J 1997; 16: 6914-6925.

4 Sun XM, Snowden RT, Skilleter DN, Dinsdale D, Ormerod MG, Cohen GM. A flowcytometric method for the separation and quantitation of normal and apoptotic thymocytes. Anal Biochem 1992; 204: 351-356.

5 Huang RF, Ho YH, Lin HL, Wei JS, Liu TZ. Folate deficiency induces a cell cycle-specific apoptosis in HepG2 cells. J Nutr 1999; 129: 25-31.

6 Allam MF, Del Castillo AS, Diaz-Molina C, Navajas RF. Invasive pulmonary aspergillosis: identification of risk factors. Scand J Infect Dis 2002; 34: 819-822.

7 Gao L, Tang CS. Cellular and molecular mechanism of cardiovascular disease resulting from hyperhomocysteinemia. Sheng Li Ke Xue Jin Zhan 2002; 33: 335-338.

8 Kokame K, Kato H, Miyata T. Homocysteine-respondent genes in vascular endothelial cells identified by differential display analysis. GRP78/BiP and novel genes. J Biol Chem 1996; 271: 29659-29665.

9 Fan G, Ma X, Wong PY, Rodrigues CM, Steer CJ. p53 dephosphorylation and p21(Cip1/ Waf1) translocation correlate with caspase-3 activation in TGF-beta1-induced apoptosis of HuH-7 cells. Apoptosis 2004; 9: 211-221. 
10 Yamaguchi Y, Shiraki K, Fuke H, Inoue T, Miyashita K, Yamanaka Y, Saitou Y, Sugimoto $\mathrm{K}$, Nakano T. Targeting of X-linked inhibitor of apoptosis protein or survivin by short interfering RNAs sensitize hepatoma cells to TNF-related apoptosis-inducing ligandand chemotherapeutic agent-induced cell death. Oncol Rep 2005; 14: 1311-1316.

11 Mercié P, Garnier O, Lascoste L, Renard M, Closse C, Durrieu F, Marit G, Boisseau RM, Belloc F. Homocysteine-thiolactone induces caspase-independent vascular endothelial cell death with apoptotic features. Apoptosis 2000; 5: 403-411.

12 Roy N, Deveraux QL, Takahashi R, Salvesen GS, Reed JC. The C-IAP-1 and C-IAP-2 proteins are direct inhibitors of specific caspases. EMBO J 1997; 16: 6914-6925.

13 Li X, Liu L, Tupper JC, Bannerman DD, Winn RK, Sebti SM, Hamilton AD, Harlan JM. Inhibition of protein geranylgeranylation and RhoA/RhoA kinase pathway induces apoptosis in human endothelial cells. J Biol Chem 2002; 277: 15309-15319.
14 Newton CJ, Ran G, Xie YX, Bilko D, Burgoyne CH, Adams I, Abidia A, McCollum PT, Atkin SL. Statin-induced apoptosis of vascular endothelial cells is blocked by dexamethasone. J Endocrinol 2002; 174: 7-16.

15 Tang D, Park HJ, Georgescu SP, Sebti SM, Hamilton AD, Galper JB. Simvastatin potentiates tumor necrosis factor alpha-mediated apoptosis of human vascular endothelial cells via the inhibition of the geranylgeranylation of RhoA. Life Sci 2006; 79: 1484-1492.

16 Taraseviciene-Stewart L, Scerbavicius R, Choe KH, Cool C, Wood K, Tuder RM, Burns $\mathrm{N}$, Kasper M, Voelkel NF. Simvastatin causes endothelial cell apoptosis and attenuates severe pulmonary hypertension. Am J Physiol Lung Cell Mol Physiol 2006; 291: L668-L676. 